\title{
Unusual behavior of the mandibular canal associated to a dentigerous cyst
}

\begin{abstract}
A dentigerous cyst is an epithelial-lined developmental odontogenic cavity that encloses the crown of an unerupted tooth at the cemento-enamel junction (CEJ). Dentigerous cysts are the second most common odontogenic cysts after radicular cysts, accounting for approximately $24 \%$ of all true cysts in the jaws. ${ }^{1-3}$ They are usually solitary in occurrence. The condition is frequently seen with the permanent dentition, usually associated with impacted mandibular third molars and maxillary canines. Pain, swelling, and facial asymmetry are occasionally seen; however, they are usually asymptomatic and observed during radiographic examination. ${ }^{4}$ This report describes a rare case of a dentigerous cyst associated with an impacted mandibular left 3rd molar with an ectopic position of the inferior alveolar canal (IAC)
\end{abstract}

Volume 2 Issue 2 - 2015

\author{
Sara Moussa, Sayde Sokhn, Ibrahim Nasseh \\ Department of Oral and Maxillo facial Imaging-School of \\ Dentistry, Lebanese University, Lebanon
}

Correspondence: Ibrahim Nasseh, Department of Oral and Maxillofacial Imaging-School of Dentistry, Lebanese University, Beirut, Lebanon, Tel 9613302232, Email ibrahim.nasseh@gmail.com

Received: November 22, 2014 | Published: April 14, 2015

Keywords: dentigerous cyst, ectopic tooth, third molar

Abbreviations: IAC, inferior alveolar canal; CT, computed tomography; CBCT. cone beam computed tomography; CEJ, cement enamel junction

\section{Introduction}

Dentigerous cyst is the most common developmental odontogenic cyst, which is believed to arise from the enlargement of the follicular space around the crown of impacted or unerupted tooth. In 1853, the term dentigerous cyst was coined by Paget. The literal meaning of dentigerous is "tooth bearing". ${ }^{5}$ One of the theories that explain the pathogeneses of this cyst is the accumulation of fluid between the unerupted tooth and the reduced enamel epithelium. The incidence of DC is two times more in males than females, where $70 \%$ occurs in the mandible and $30 \%$ in the maxilla. ${ }^{5}$ Although a DC may be associated with any unerupted tooth but the mandibular third molar and maxillary canine are the most involved followed by mandibular premolar and maxillary third molar. ${ }^{5}$ Dentigerous cyst is discovered by routine radiographs or when radiographs are taken to investigate the failure of a tooth eruption, a missing tooth or misalignment. No pain or discomfort is present with the cyst unless its secondary infected. Radiographically, the cyst appear well defined (unless infected), unilocular radiolucency associated with the crown of unerupted tooth. The involved tooth can be displaced away from its path of eruption. While a normal follicular space is $3-4 \mathrm{~mm}$, a DC is suspected when the space is more than $5 \mathrm{~mm} .^{1}$ The classical treatment of DC is enucleation and extraction of the involved tooth. ${ }^{6}$ The present paper reports an additional case of DC with an unusual behavior of the IAC.

\section{Case report}

A 55-years-old male presented to our department, with a chief complain of severe pain in the mandibular left molar region. Past medical and dental history revealed that approximately one year ago the patient complained of pain at the same region, where defective endodontic treatment was performed on tooth \#36 in a private dental clinic but no permanent relief was obtained. No trauma history was reported. On extra oral inspection, there was a swelling on the lower left side. Intraoral examination showed obliteration of the left labial vestibule compared to the right premolar molar area. Teeth \#36-37 showed no pain on percussion. Absence of the wisdom tooth \#38 was noticed. The panoramic examination revealed a well-defined, corticated unilocular radiolucent area associated with an inverted tooth \#38, located near the angle of the mandible, occupying the whole height of the ramus from the upper border of the mandible till the lower border, with a marked thinning of the borders. The inferior alveolar canal (IAC) is remarkably non visible at the region of the lesion (Figure 1).

A CBCT was performed to identify the extension of the lesion and its relation with the IAC. Panoramic reconstruction (Figure 2A \& 2B) shows a well-defined low density unilocular lesion circumscribing the crown of tooth \#38 located vertically from the mid ramus extending below the level of the IAC, horizontally it extend from the apical third of the mesial root of \#37 till the mid portion of the ascending ramus. Axial cuts (Figure 3) show expansion of the alveolar process and thinning and interruption of both lingual and buccal cortical plates. The roots of \#37 show no resorption. The lesion is attached to tooth \#38 at the level of the CEJ (Figure 4). The ectopic position of the $\mathrm{IAC}$ is also seen very clearly in the maximum intensity projection panoramic reconstruction (Figure $2 \mathrm{~B}$ ) and in the cross sectional images which shows the canal within the lesion (Figure 5A \& 5B). After clinical and radiographic examination, a provisional diagnosis of dentigerous cyst was made; however, kerato cyst odontogenic tumor, amleloblastoma, ameloblastic fibroma were also considered in the differential diagnosis. Keratocyst don't expand the bone to the same degree as presented here and attachment to the teeth will be more apically. Ameloblastic fibroma usually occurs in young patients which is not the case. Ameloblastoma would have presented internal septa if big in size as in this case. The most probable diagnosis is dentigerous cyst. The operation was performed under local anesthesia. Incision was made followed by raising a full thickness buccal mucoperiosteal flap. The lesion was then identified and the canal was viewed and confirmed, as seen on CBCT, to be passing in the center of the lesion. 
Dissection of the lesion away from the canal was performed using a tissue holder. With proper identification and isolation, the inferior nerve was preserved and the cyst was totally enucleated. Tooth number \#38 was removed, and preservation of tooth \#37. No sign of paresthesia was recorded after surgery. The surgical specimen was then sent for pathological analysis for final diagnosis. The histological examination showed a cystic lumen with blood content, a very thin lining of non-keratinized stratified squamous epithelium and dense fibro cellular connective tissue stroma with chronic inflammatory infiltrate. A macrophage and giant cell granuloma is noted around cholesterol crystals. There is no evidence of malignant change. The cyst opened measured $4 \times 2 \times 1 \mathrm{~cm}$. A chronic inflamed dentigerous cyst was confirmed. Follow up CBCT was done after 3years of the surgery, and a progressive improvement in bone regeneration and healing process at the site of surgery was observed (Figure 6A \& 6B); vitality test on tooth $\# 37$ response was positive.

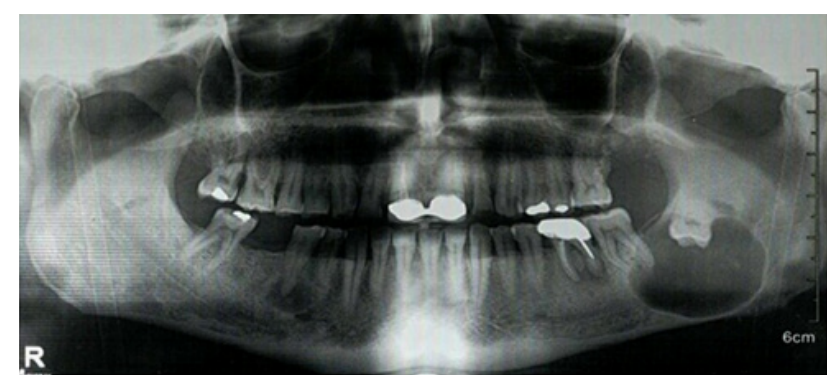

Figure I Pre-operative panoramic x-ray showing a well-defined unilocular radiolucency associated with tooth \#38, near the angle of the mandible.

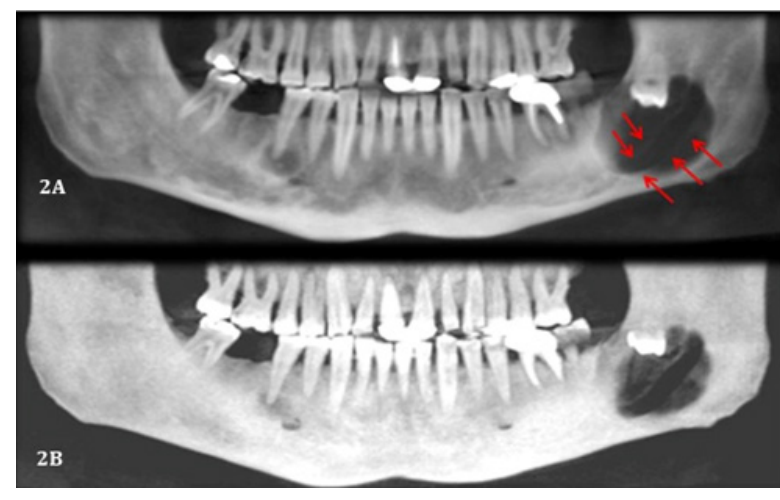

Figure 2A Panoramic reconstruction showing the ectopic position of the IAC. Figure 2B Maximum intensity projection confirming the IAC position.

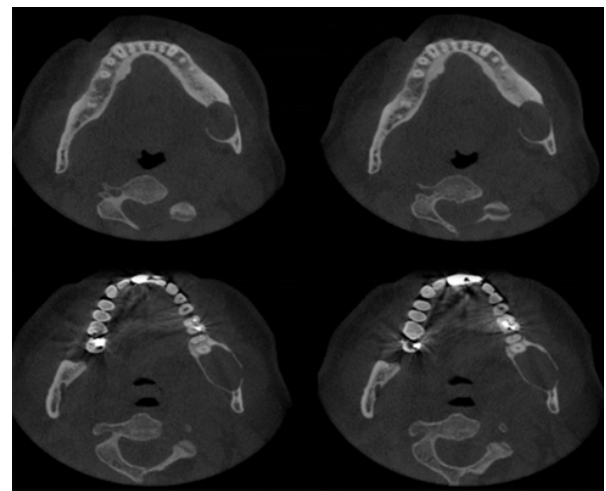

Figure 3 Axial cuts ( $1.2 \mathrm{~mm}$ interval) at the level of mid root showing of both lingual and buccal cortical plates and interruption of the buccal lingual cortical plates at different levels; marked expansion of the cortical plate is also noted.

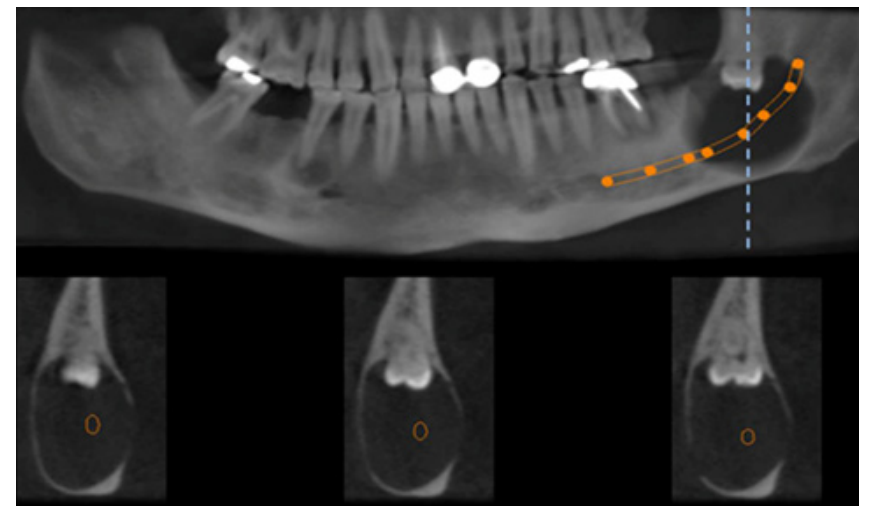

Figure 4 Frontal cut showing the lesion starting at the cement-ename junction.

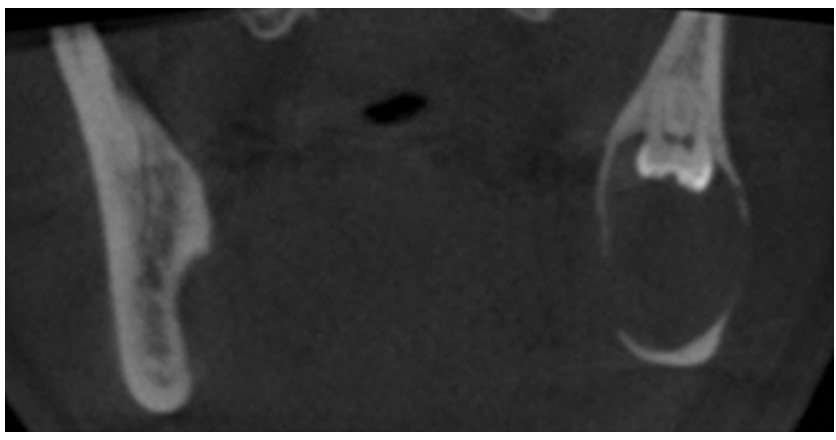

Figure 5A Panoramic reconstruction with mandibular canal tracing; cross sectional images showing the position of the IAC within the lesion.

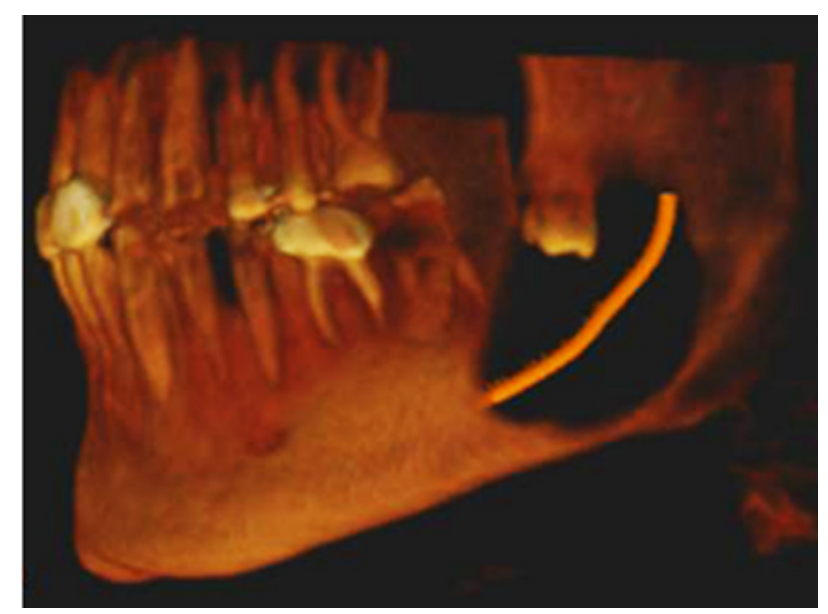

Figure 5B 3D reconstruction showing the lesion and the position of the IAC.

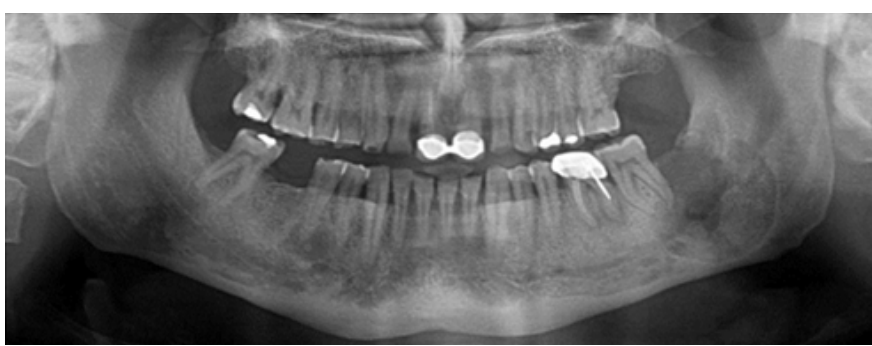

Figure 6 (A) Post-op panoramic reconstruction with cross sectional images showing the healing process after 3 years from the surgery and the position of the canal. 


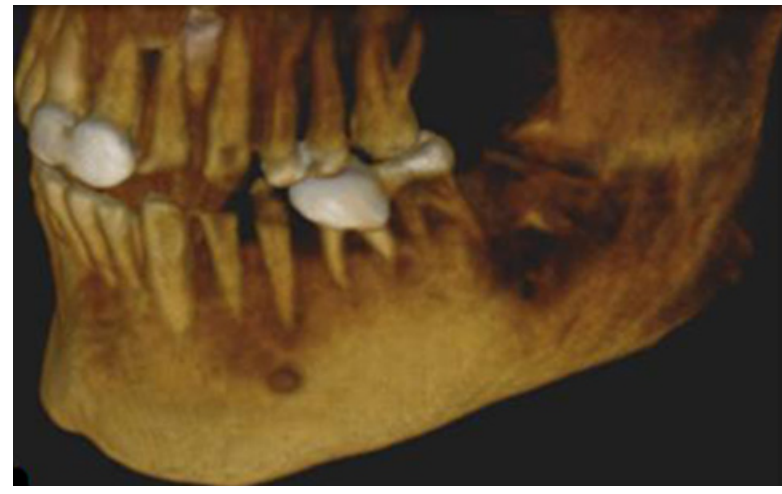

Figure 6 (B) Post-op 3D reconstruction showing bone regeneration.

\section{Discussion}

Pathologies such as cyst or tumor in relation to a developing tooth is one of the three main causes of ectopic eruption of the tooth, the others being-developmental disturbance and iatrogenic activity. ${ }^{7} \mathrm{~A}$ dentigerous cyst is defined as a cyst that encloses the crown of an unerupted tooth, expands the follicle and is attached to the cement enamel junction of the unerupted tooth. The substantial majority of dentigerous cysts involve the mandibular third molar and the maxillary permanent canine, followed by the mandibular premolars, maxillary third molars and rarely the maxillary premolars. Studies have shown that the incidence rate of dentigerous cysts involving the maxillary premolar was $2.7 \%$ as compared to $45.7 \%$ involving the mandibular third molar. Mourshed stated that $1.44 \%$ of impacted teeth undergo dentigerous cyst transformation. ${ }^{1}$ Dentigerous cysts most commonly occur in the 2nd and 3rd decades of life. ${ }^{1,8}$ Dentigerous cysts can reach considerable size with minimal or no symptoms, early detection and removal of the cysts is important to reduce morbidity. Most dentigerous cysts are usually solitary, slow growing, completely asymptomatic and remain dormant until infected or until they have enlarged substantially, producing swelling, expansion and thinning of the cortex which may lead to pathological fracture of the jaw.

The infection occurs after severe thinning and interruption of the cortical outline followed by interruption of the cyst epithelial lining and communication between the cyst lumen of and the oral cavity.

Conventional radiographs are useful in identifying and establishing a provisional diagnosis of lesions. But specialized imaging modalities like computed tomography (CT) and cone beam computed tomography (CBCT) are of most importance in the management of these cysts which are in close relation with vital anatomical structure. CBCT is especially valuable in demarcating the hard tissue periphery from the soft tissue involvement of the lesion. The major advantages of CBCT are that they provide multiplanar reformation with volume reconstructions and $3 \mathrm{D}$ images with a much lower radiation dose when compared to computed tomography. As a result CBCT enable the surgeon to accurately assess the extent of the lesion as well as its proximity to the contiguous vital structures. ${ }^{3}$

In our case, a CBCT was made after primary investigation of the case by panoramic radiograph to show the exact extend of the lesion. Panoramic reconstruction showed a well-defined corticated unilocular homogeneous low density lesion associated with the crown of the unerupted inverted wisdom tooth \#38, occupying the angle of the mandible. The lesion shows bucco-lingual expansion and interruption of cortical plates, no root resorption on the adjacent tooth (\#37) and displacement of the affected tooth (\#38) in coronal direction. In relation with the mandibular canal, there was no remarkable change in the position, which contradicts the benign aspect of the lesion and its slow growing rate. Slowly growing lesions will have adequate time for remodeling and subsequent pushing of the canal. This was not present in our case, whereas the lesion continued its slow growing process around the mandibular nerve, without any change in its position and without any clinical signs.

\section{Conclusion}

Dentigerous cyst is a benign slowly growing odontogenic cyst that may reach large size affecting the mandibular body and ramus, and even the whole antrum without any chief complaint from the patient unless it is infected; therefore it is essential to perform radiographic examination of unerupted teeth. Usually conventional radiography fail to delineate the full extent of a lesion if present, so performing more advanced imaging such as CBCT may permit a better delineation of the extent of the lesion and its relation to the adjacent anatomical structures. The case presented showed us the importance of CBCT in marking the ectopic position of the IAC in relation to a slowly growing dentigerous cyst, and the exact extent of the lesion and its expansion, all this information gathered will aid the surgeon in his pre-surgical planning to prevent blinded surgery with its consequence complications.

\section{Acknowledgement}

None.

\section{Funding}

None.

\section{Conflicts of interest}

The authors declare that there is no conflict of interest.

\section{References}

1. Tamgadge A, Tamgadge S, Bhatt D, et al. Bilateral dentigerous cyst in a non-syndromic patient: Report of an unusual case with review of the literature. J Oral Maxillofac Pathol. 2011;15(1):91-95.

2. Saluja JS, Ramakrishnan MJ, Vinit GB, et al. Multiple dentigerous cysts in a nonsyndromic minor patient: Report of an unusual case. Natl J Maxillofac Surg. 2010;1(2):168-172.

3. Shafer H, Levy. Cyst and tumors of odontogenic origin in. Shafers Textbook of oral pathology, $5^{\text {th }}$ ed. India: Elsevier; 2007. pp. 358-361.

4. Hasan S, Ahmed SA, Reddy LB. Dentigerous cyst in association with impacted inverted mesiodens: Report of a rare case with a brief review of literature. Int J Appl Basic Med Res. 2014;4(S1):S61-S64.

5. Kasat VO, Karjodkar FR, Laddha RS. Dentigerous cyst associated with an ectopic third molar in the maxillary sinus: A case report and review of literature. Contemp Clin Dent. 2012;3(3):373-376.

6. Carrera M, Dantas DB, Marchionni AM, et al. Conservative treatment of the dentigerous cyst: report of two cases. Braz J Oral Sci. 2013;12(1).

7. NSM, Krishnamoorthy B, JKS, et al. Diagnostic CBCT in Dentigerous Cyst with Ectopic Third Molar in the Maxillary Sinus-A Case Report. $J$ Clin Diagn Res. 2014;8(6):ZD07-ZD09.

8. Shear M, Speight PM. Cyst of oral and maxillofacial regions. $4^{\text {th }}$ ed. USA: Blackwell publishers; 2007. pp. 59-75. 